März bis 17. April 2015 erneut krank war, mochte der Arbeitgeber nicht mehr glauben, dass wiederum eine neue Krankheit vorliegt. Auf Nachfrage be scheinigte die Krankenkasse aber genau dies. Doch damit gab sich der Arbeitgeber nicht zufrieden. Die Ärzte hätten die Frau wohl nicht richtig untersucht oder hätten irrtümlich verkannt, dass es sich um eine Fortsetzungserkrankung handelt. Daher müsse die Gruppenleiterin die Diagnosen ihrer AU-Bescheinigungen offenlegen, damit er dies überprüfen könne.

Die Gruppenleiterin entband ihre Ärzte daraufhin teilweise von der Schweigepflicht, sodass diese bescheinigen konnten, dass die neue Erkrankung nicht auf einem der früheren Grundleiden beruht. Die konkreten Diagnosen erhielt der Arbeitgeber aber nicht. Er verweigerte daher die Lohnfortzahlung. Das LAG gab in dem Streit nun der Arbeitnehmerin recht. Die BAG-Rechtsprechung aus 2005 sei widersprüchlich. Zur Begründung verwies das LAG auf Fälle, in denen die Krankenkasse Krankengeld gezahlt hat. Wenn dennoch Anspruch auf Entgeltfortzahlung bestand, geht dieser Anspruch insoweit auf die Krankenkasse über. Dabei darf sie die Diagnosen aber nicht mitteilen, weil diese dem Sozialdatenschutz unterliegen.

\section{Revision zugelassen}

Um Ungleichbehandlungen zu vermeiden, müssten sich auch die Arbeitnehmer selbst auf den Sozialdatenschutz berufen können, argumentierte das LAG. Es sei nicht einsichtig, dass Arbeitgeber ihre Mitarbeiter zur Offenlegung von Daten zwingen könnten, „die die Krankenkasse nicht hätte offenbaren dürfen“. Ähnlich wie beim Beweiswert einer AUBescheinigung selbst müsse daher der Arbeitnehmer die Diagnosen zumindest so lange zurückhalten können, „bis der Arbeitgeber Tatsachen vorlegt, die zu Zweifeln an der Richtigkeit der Mitteilung der Krankenkasse Anlass geben“. Im konkreten Fall habe der Arbeitgeber dies nicht getan.

Wegen seiner Abweichung von der höchstrichterlichen Rechtsprechung des BAG ließ das LAG die Revision zu. $\mathrm{Ob}$ der Arbeitgeber sie einlegt, ist derzeit noch offen. Martin Wortmann

\title{
eGK: kein Recht auf „analoge Welt"
}

Die Einführung der elektronischen Gesundheitskarte ist grundsätzlich rechtmäßig, jedoch darf der Datenschutz nicht durch die Speicherung verschiedener Zusatzinformationen zum "Versichertenstatus" unterlaufen werden. Das Landessozialgericht Baden-Württemberg hat hier entsprechend entschieden.

$B^{c}$ ereits 2014 hatte das Bundessozialgericht in Kassel entschieden, dass die elektronische Gesundheitskarte (eGK) mit Lichtbild des Versicherten und Datenchip nicht gegen das Recht auf informationelle Selbstbestimmung verstößt (Az.: B 1 KR 35/13 R). Dem schloss sich das Landessozialgericht Stuttgart nun an. Das Grundrecht auf informationelle Selbstbestimmung gewähre „kein Recht auf Verhinderung der Digitalisierung und Weiterleben in einer analogen Welt“. Versicherte müssten die Gesundheitskarte daher ihrem Arzt vorlegen. Der Einzelne habe „kein Recht im Sinne einer absoluten, uneinschränkbaren Herrschaft über ,seine‘ Daten“". Mit der Kas- senärztlichen Bundesvereinigung hatten die gesetzlichen Krankenkassen allerdings vereinbart, dass ohne Zustimmung der Versicherten zusätzliche „statusergänzende Merkmale“ zum Versichertenstatus gespeichert werden sollen. Dazu gehört die Teilnahme an DMPProgrammen und der „Ambulanten Spezialfachärztlichen Versorgung“ (ASV). Nach Überzeugung des Landessozialgerichts Stuttgart ist das von den gesetzlichen Vorgaben nicht abgedeckt. Der Begriff des Versichertenstatus dürfe nicht „beliebig ausgefüllt und datenmäßig erweitert" werden, so die Richter (Az.: L 11 KR 2510/15).

Martin Wortmann
Berufshaftpflichtpolice: Angestellte Ärzte sind für Fiskus tabu

Keine zusätzlichen Lohnsteuern und Sozialversicherungsbeiträge müssen angestellte Ärzte einer Gemeinschaftspraxis zahlen, nur weil diese für sich als Personengesellschaft eine Berufshaftpflichtversicherung abgeschlossen hat. Finanzämter dürfen die Versicherungsbeiträge der Personengesellschaft nicht so behandeln, als ob sie bei den Arbeitnehmern zu zusätzlichem Arbeitslohn führen würden. Dieses Verbot gilt selbst dann, wenn der Versicherungsschutz auch Ansprüche gegen die Angestellten abdeckt. Das hat der Bundesfinanzhof mit Aktenzeichen VI-R-58/14 entschieden. Julia Frisch

\section{Fundraising bringt Kliniken zusätzliche Mittel ein}

Fundraising ist für Krankenhäuser ein erfolgversprechendes Modell. Bereits über $60 \%$ aller Kliniken in Deutschland mit mehr als 100 Krankenbetten haben laut einer aktuellen Studie der Unternehmensberatung Roland Berger bereits erfolgreich Spenden eingeworben. Die Durchschnittseinnahmen lägen bei knapp einer halben Million € pro Krankenhaus - Tendenz steigend. Zusätzlich verschaffe das Fundraising den Kliniken positive Nebeneffekte wie eine gute AuBendarstellung. Diese mache das Spendeneinwerben für die Häuser umso attraktiver.

Matthias Wallenfels

\section{Bereits $\mathbf{5 0 0}$ Gesundheits-Apps getestet}

Die Testdatenbank von HealthOn, nach eigenen Angaben die größte unabhängige Plattform für Gesundheits-Apps in Europa, hat die Summe von 500 Einträgen erreicht. Das teilt die Organisation mit und benennt den 500 . Testbericht als "wichtigen Meilenstein“. Das Internetportal hat sich zum Ziel gesetzt, unter anderem Ärzten dabei zu helfen, die Güte von Apps zu beurteilen. Dabei unterstütze das Portal Anwender mit einer Checkliste. Momentan stünden deutschsprachigen Verbrauchern insgesamt 10.000 Gesundheits- und Medizin-Apps zur Verfügung. Nur Apps, die umfassend informieren und den Verbraucherschutz ernst nehmen, verdienen das Vertrauen der Nutzer, heißt es.

Matthias Wallenfels 